\title{
Free Health Coverage for All COVID-19 Patients is Required to Defeat the Virus at The Community Level
}

Rafiye Ciftciler ${ }^{1}$, [MD]

ORCID: 0000-0001-5687-8531

Ibrahim Celalettin Haznedaroglu' ${ }^{1}$, [MD]

ORCID: 0000-0001-8028-9462
${ }^{1}$ Hacettepe University, Faculty of Medicine, Department of Hematology, Ankara, Turkey.

Corresponding Author: Rafiye Ciftciler Departments of Hematology, Hacettepe University Faculty of Medicine, Ankara, Turkey.

E-mail: rafiyesarigul@gmail.com

https://doi.org/10.32552/2021.ActaMedica.562

Received: 2 February 2021, Accepted: 17 February 2021, Published online: 5 June 2021
Our country, Turkey, brilliantly outlined the preventive measures for COVID-19, to be taken across the country to restrict the ongoing viral spread via following the pathways of science [1]. On the other hand, several journal Editors inaugurated historical evidences to outline master management deficiencies in other countries during the current complicating course of the pandemic [2]. We would like to point out another challenging issue in many countries, that is the individual COVID-19 healthcare costs and high individual co-payments for numerous people even in patients having substantial level of health insurance. Those socio-economical facts further pronounced in the upcoming news regarding the development of vaccines against SARS-CoV-2 infection. The vaccines and the entire clinical COVID-19 care shall be freely available not only for 'the rich' but also for 'the poor' in order to establish a wide "immunity barrier" to stop current pandemic disaster.

Our country, Turkey, has significantly lowercase fatality ratios (2.8\%; and 52.5 infections/million population) in comparison to many Western countries as officially acknowledged by World Health Organization (WHO) [3]. Free health coverage for the diagnosis and treatment of patients infected with SARSCoV-2 were made free of charge for all citizens and residents of Turkey. The completely free clinical healthcare of Turkish patients already represented as a lesson for universal coverage [4]. The vaccination for SARS-CoV-2 also will be free for every Turkish people regardless of their level of insurance.

We, as the experts in chronic myeloid leukemia (CML), had previously declared scientific and ethical aspects of free, or at least acceptable, coverage of individual healthcare costs in CML [5]. The quote of "The best tolerated pain is the other person's pain", which is unfortunately working in cancer and other noninfectious diseases, is not valid in COVID-19 case since the virus in the poor could hit the body of the richest as well. Poorly eradicated SARS-CoV-2 virus in the poor man may convert him to a super-spreader, which then affect the rich man within the same community. Therefore, currently ongoing pandemic crisis further indicated the fact that free health coverage for all COVID-19 patients is absolutely required to defeat the virus at the large community level. It is hoped that humanity and science cooperate for this critical issue to stop this pandemic evil. 


\section{CONFLICT Of INTEREST}

No benefits in any form have been received or will be received from a commercial party related directly or indirectly to the subject of this article.

\section{we REFERENCES CQu}

[1] Çiftçiler R, Haznedaroglu IC, Tufan A, et al. COVID-19 Scientific Publications From Turkey. Turk J Med Sci. 2020.

[2] The Editors. Dying in a Leadership Vacuum. N Engl J Med. 2020;383(15):1479-80.

[3] World Health Organization (WHO).Turkey's response to COVID-19: first impressions. Ankara, Turkey. 11 July 2020. Copenhagen: WHO Regional Office for Europe; 2020. Licence: CC BY-NC-SA 3.0 IGO: World Health Organization. Regional Office for Europe; 2020:1-25.

[4] Atun R. Transforming Turkey's Health System-Lessons for Universal Coverage. N Engl J Med. 2015;373(14):1285-9.

[5] Expert in Chronic Myeloid Leukemia. The price of drugs for chronic myeloid leukemia (CML) is a reflection of the unsustainable prices of cancer drugs: from the perspective of a large group of CML experts. Blood. 2013;121(22):4439-42. 